\title{
Télescope
}

Revue d'analyse comparée en administration publique

\section{L'audit de la gouvernance : au service d'une plus grande transparence}

\section{Normand Ouellet}

Volume 18, numéro 3, automne 2012

URI : https://id.erudit.org/iderudit/1013780ar

DOI : https://doi.org/10.7202/1013780ar

Aller au sommaire du numéro

Éditeur(s)

L’Observatoire de l'administration publique

ISSN

1203-3294 (imprimé)

1929-3348 (numérique)

Découvrir la revue

Citer cet article

Ouellet, N. (2012). L'audit de la gouvernance : au service d'une plus grande transparence. Télescope, 18(3), 157-171. https://doi.org/10.7202/1013780ar
Résumé de l'article

Cet article met en lumière les différents enjeux qui font en sorte que l'audit gouvernemental, ou de la gouvernance, pourrait être appelé à revêtir de plus en plus d'importance compte tenu de l'engagement clair pour un gouvernement plus transparent et du vecteur de changement sans précédent que constitue le développement exponentiel des moyens inter- actifs de communication. Il propose une réflexion prospective sur l'évolution de la fonction d'audit gouvernemental dans le contexte d'une plus grande ouverture des gouvernements à la participation et à la collaboration des citoyens. 


\title{
L'AUDIT DE LA GOUVERNANCE : AU SERVICE D'UNE PLUS GRANDE TRANSPARENCE
}

\author{
Par Normand Ouellet ${ }^{1}$, Expert en audit et Candidat au doctorat en santé des \\ populations, Université d'Ottawa・nouel056@uottawa.ca
}

\begin{abstract}
RÉSUMÉ Cet article met en lumière les différents enjeux qui font en sorte que l'audit gouvernemental, ou de la gouvernance, pourrait être appelé à revêtir de plus en plus d'importance compte tenu de l'engagement clair pour un gouvernement plus transparent et du vecteur de changement sans précédent que constitue le développement exponentiel des moyens interactifs de communication. Il propose une réflexion prospective sur l'évolution de la fonction d'audit gouvernemental dans le contexte d'une plus grande ouverture des gouvernements à la participation et à la collaboration des citoyens.
\end{abstract}

\begin{abstract}
This article highlights the various issues involved in driving a potential greater role for governmental audit or audit of governance. Such drivers include the clear commitment to greater openness on the part of governments and the exponential development of interactive modes of communication. In fact, this text proposes a prospective reflection on the emerging role of governmental audit in the context of greater receptiveness by governments to the participation and collaboration of citizens.
\end{abstract}

Pour citer cet article: Ouellet, N. (2012). "L'audit de la gouvernance : au service d'une plus grande transparence », Télescope, vol. 18, n³, p. 157-171.

vec la venue des médias sociaux, la capacité de communiquer de l'informaA tion selon des modes interactifs constitue un vecteur de changement sans précédent qui a des incidences sur la gouvernance de nos sociétés. La facilité d'interaction que procurent les médias sociaux représente en soi une opportunité et un potentiel encore inexploité. Cette facilité favorise l'exercice d'une démocratie participative misant sur davantage de transparence et sur une obligation de reddition de comptes bonifiée de la part des leaders politiques. Dans le présent texte, nous émettons l'hypothèse qu'une plus grande participation des citoyens à la gestion du bien commun induit un plus grand degré de transparence et facilite ainsi une meilleure reddition de comptes des acteurs politiques et des institutions publiques en général. La fonction d'audit est utile à la gouvernance. Si cette gouvernance évolue vers une plus grande participation citoyenne, la fonction d'audit aura de nouveaux défis à relever en soutenant cette nouvelle dynamique plus interactive entre les citoyens et leurs institutions publiques.

Du temps de la période absolutiste, le roi n'avait pas le devoir de rendre compte de sa gestion. C'étaient plutôt ses subordonnés qui avaient, et qui ont encore aujourd'hui, l'obligation de rendre des comptes à l'autorité. De nos jours, les

\footnotetext{
1 L'auteur tient à remercier la contribution importante que les pairs ont apportée lors de la revue de I'article avant sa publication. Cette revue a permis d'améliorer substantiellement le présent texte.
} 
citoyens revendiquent une plus grande transparence de la part des acteurs publics et politiques. La participation et la collaboration des citoyens à une démocratie plus participative nous amènent d'une dynamique unidirectionnelle à une dynamique bidirectionnelle et interactive où l'autorité exécutive aura de plus en plus à rendre des comptes.

Le conte Les habits neufs de l'empereur (Anderson, 1837) est révélateur des prétentions de la transparence dans les rapports d'autorité hiérarchique fortement axés du haut vers le bas. Selon ce conte, alors que l'empereur ainsi que tous ses sujets se mentent à eux-mêmes et forcément aux autres, seul l'enfant humble préserve les véritables qualités de la transparence en déclarant publiquement la nudité de l'empereur. L'empereur n'a pas pour autant modifié son parcours. D'ailleurs, ses proches collaborateurs, même ceux se prétendant indépendants et objectifs, ont préféré ignorer et nier l'évidence afin de maintenir leurs prérogatives relationnelles avec le pouvoir. Pour que la gouvernance collaborative s'épanouisse, il est nécessaire que l'ensemble des acteurs sociaux collabore à la gestion publique selon une dynamique relationnelle respectueuse empreinte de rapports égalitaires. La vision hiérarchique supporte bien le caractère monarchique de nos sociétés, mais représente souvent un frein au processus de démocratisation et à une plus grande transparence.

Les bureaucraties des États sont caractérisées par un type d'autorité fondamentalement hiérarchique et descendant. Ce type d'autorité donne lieu à une diffusion d'information unidirectionnelle depuis l'appareil étatique jusqu'aux citoyens où la vision et les référents de la communication sont définis par le pouvoir politique et son impressionnante armada bureaucratique. À l'instar de l'entourage de l'empereur nu du conte d'Anderson, au Canada et au Québec, une armada subordonnée au pouvoir politique maintient le statu quo pour préserver ses prérogatives relationnelles avec le parti politique formant le gouvernement majoritaire qui contrôle les pouvoirs exécutif et législatif et, par conséquent, les deniers publics.

En ce début de millénaire, l'ordre du jour est à une démocratie plus directe. De nombreux pays se sont engagés dans l'objectif du gouvernement ouvert et se sont regroupés dans l'Open Government Partnership (2011) (Partenariat pour un gouvernement transparent). Qui aurait cru à l'époque absolutiste que le roi devrait rendre des comptes au peuple! La déclaration du président Obama déposée au début de son mandat en janvier 2009 résume bien l'esprit qui anime ce partenariat :

My Administration is committed to creating an unprecedented level of openness in Government. We will work together to ensure the public trust and establish a system of transparency, public participation, and collaboration. Openness will strengthen our democracy and promote efficiency and effectiveness in Government. (Obama, 2009)

Selon les principes de l'Open Government Partnership, toutes les informations gouvernementales accessibles aux citoyens devraient être mises en ligne. Un meilleur accès à l'information signifie un degré plus élevé de transparence et favorise la participation citoyenne aux débats publics et une meilleure reddition de comptes de l'appareil d'État. Certes, un long chemin reste à parcourir pour concrétiser l'engagement de l'Open Governement Partnership et l'utilisation généralisée 
d'Internet et des médias sociaux fait en sorte que les attentes des citoyens pour une plus vaste transparence augmentent de façon exponentielle.

Dans le texte qui suit, un tour d'horizon du rôle de l'audit gouvernemental est d'abord présenté pour ensuite aborder les enjeux importants qui conditionneront son évolution vers une fonction d'audit qui s'inscrit dans une gouvernance collaborative au service d'une démocratie plus participative. Les enjeux liés à l'engagement des quarante-sept pays - dont le Canada - à l'endroit de l'Open Government Partnership sont clairement énoncés dans la déclaration du président Obama : confiance du public, ouverture et transparence, participation et collaboration citoyennes et efficience et efficacité du gouvernement (Obama, 2009). L'audit gouvernemental devra s'adapter et évoluer dans le contexte d'une reddition de comptes électronique, interactive et très rapidement accessible.

\section{- UN TOUR D'HORIZON DU RÔLE DE L'AUDIT GOUVERNEMENTAL}

La fonction d'audit est utile à la gouvernance, car elle soutient l'autorité dans la délégation de responsabilités en venant certifier la qualité de la reddition de comptes. Elle offre donc une garantie aux récepteurs du message (les citoyens, les actionnaires ou toutes parties déléguant pouvoir, autorité et responsabilité) quant à la fiabilité de l'information communiquée. En théorie, l'audit sert à maintenir ou à raffermir le lien de confiance entre l'émetteur et le récepteur de la reddition de comptes. Ce récepteur peut être rassuré par un audit, une évaluation ou une enquête venant lui offrir une opinion indépendante et objective sur le message communiqué lors de la reddition de comptes, sur la bonne conduite des affaires selon les pratiques exemplaires généralement reconnues ou encore sur la saine gestion des risques d'affaires et organisationnels.

Divers mécanismes de reddition de comptes permettent de communiquer de l'information pour satisfaire aux attentes de transparence. Compte tenu des perspectives et des intérêts différents des acteurs, des services d'assurance sont apparus pour venir certifier de façon indépendante et objective la qualité de l'information communiquée selon un cadre normatif de présentation établi. Ces services sont importants pour maintenir la confiance en l'intégrité de l'information fournie.

La tradition d'audit s'est développée dans le terreau du monde de la finance. À partir d'un cadre normatif de présentation de l'information financière, les banquiers ont exigé de leurs clients emprunteurs qu'ils rendent des comptes sur leur rendement financier et qu'ils obtiennent une opinion indépendante et objective d'auditeurs venant confirmer la qualité des informations. Le besoin d'assurance des actionnaires de grandes entreprises et des banquiers a permis de mettre en place un modèle d'audit de l'information financière et de développer les normes internationales d'information financière ainsi que la méthodologie et les normes internationales d'audit.

L'obligation de rendre des comptes est la contrepartie essentielle découlant de la délégation de pouvoir. Des liens de confiance doivent être maintenus ou raffermis à chacun des paliers de délégation, soit : 
- entre les citoyens et le Parlement (les députés) (niveau 1);

- entre le Parlement (les députés - pouvoir législatif) et le gouvernement (le cabinet ministériel - pouvoir exécutif) (niveau 2);

- entre le gouvernement et les administrateurs publics (niveau 3);

- entre les administrateurs publics et les gestionnaires publics (niveau 4).

Les assises de l'audit gouvernemental sont bien établies dans la délégation des niveaux 3 et 4, notamment avec les services d'audit interne, et dans la délégation de niveau 2 , notamment avec le vérificateur général.

Toutefois, en ce qui concerne le premier niveau de délégation, c'est-à-dire celui entre les citoyens et leurs représentants formant le Parlement, il n'y a pour l'instant aucun service d'audit en place. Les élections, mécanisme fondamental de notre démocratie, sont actuellement le seul mécanisme direct par lequel les citoyens posent un geste de participation à la vie politique qui vient témoigner de leur confiance. Le développement d'une relation interactive entre les citoyens et l'appareil d'État pourrait être facilité par la mise en place d'un processus d'audit de la gouvernance.

L'application du modèle d'audit financier au secteur public a évolué pour prendre en compte l'étendue des besoins d'assurance des parties prenantes. Certes, les états financiers des gouvernements (les comptes publics) font l'objet d'un audit qui permet d'offrir une assurance quant à la fiabilité des informations sur leur rendement financier, mais la performance des administrations publiques ne se limite pas à la dimension de surplus ou de déficit budgétaire. L'audit gouvernemental a ouvert la voie à l'audit de la performance organisationnelle.

Afin de combler les besoins d'assurance liés à la reddition de comptes découlant de diverses délégations de pouvoir et de tenir compte d'indicateurs de performance intégrant l'ensemble des objectifs des administrations publiques, la notion d'audit intégré est apparue pour, théoriquement, certifier l'efficacité, l'efficience, l'économie et, plus récemment, le développement durable, l'éthique et la saine gouvernance. Une question fondamentale se pose : l'intérêt des citoyens est-il pris en compte et les citoyens en ont-ils vraiment pour leur argent? On parle désormais d'audit de l'optimisation des ressources, d'audit de la performance, d'audit interne pour les besoins d'assurance des acteurs de gouverne, etc. Quelle que soit l'appellation (audit, évaluation, inspection, enquête), l'objectif demeure d'émettre, à partir d'un référentiel normatif qui précise les objectifs et les critères d'audit, une opinion et des recommandations, le cas échéant, visant à rassurer les parties prenantes au sujet du rendement et de l'atteinte des résultats. L'administration publique et le gouvernement font appel à plusieurs acteurs de surveillance de leurs activités qui devraient rassurer les récepteurs de la reddition de comptes au sujet de l'information communiquée quant aux résultats obtenus ou attendus.

Le Parlement s'est doté de commissions parlementaires sectorielles, de mécanismes de commission d'enquête, de plusieurs agents du Parlement comme le vérificateur général et le protecteur du citoyen. De plus, les acteurs de gouverne de chaque ministère, organisme et société d'État ont accès à des services d'audit interne, d'enquête et d'évaluation de programme. Tous ces services de surveillance soutiennent les acteurs de gouverne (parties déléguant pouvoir et responsabilité) 
et fournissent des services ayant comme fonction d'offrir une garantie quant à la bonne marche des affaires.

\section{- LA CONFIANCE DU PUBLIC : LA CLÉ DE VOÛTE RELATIONNELLE INDISPENSABLE}

En fonction de leur culture et de leurs divers intérêts, les acteurs reçoivent et envoient de l'information de manière sélective. Cette sélectivité vient en quelque sorte tamiser ou colorer la clarté ou la transparence qui dépeint une version toujours limitée de la réalité ou de la vérité. Nul doute que l'empirisme apporte une contribution à la démarche scientifique. Toutefois, divers courants épistémologiques, tel le constructivisme, suscitent des réserves quant à l'interprétation et aux tentatives intuitives d'explication de la réalité à partir d'un ensemble de faits ou de modèles (voir à cet égard Riopel, 2009). L'acteur, en raison de sa dimension politique, fait parler les faits selon ses perceptions toujours sélectives. Cette sélectivité dans la façon de positionner une situation ou un problème ou d'établir un jugement ou un constat n'est jamais totalement dénuée d'intérêt. De plus, elle est fortement conditionnée par notre culture, soit par notre façon de vivre, de ressentir et de penser. Notre culture est vivante et évolutive et donc en perpétuel changement. Tout comme le latin est disparu pour former d'autres langues, le processus d'acculturation vient du caractère changeant et évolutif de l'environnement et fait en sorte que de nouveaux paradigmes se créent.

L'ère télévisuelle de masse cède tranquillement le pas à l'ère du Web interactif. Les nouvelles facilités de communication interactive et instantanée ont le potentiel d'ouvrir le chemin à une plus grande participation citoyenne dans la gestion publique et politique. Dans les prochaines décennies, des représentants de la génération des enfants nés avec une souris d'ordinateur dans les mains et friands de jeux interactifs et des médias sociaux seront nos leaders et auront les mains sur le gouvernail de l'État!

Si nous admettons que nous faisons constamment parler les faits en fonction notamment d'intérêts ou d'idéologies, il devient naturel de remettre en question les propositions d'interprétation de la réalité construites selon des perspectives rarement totalement neutres et objectives. Parce que l'être humain ne peut se dissocier totalement de son environnement, sa subjectivité façonne sa vision du monde. Nous devons donc être pragmatiques et abandonner les absolus pour composer avec des degrés d'objectivité, d'indépendance ou de transparence. Dans ce contexte, la confiance entre les acteurs risque de se brouiller, car les messages sont toujours enrobés de couleurs offrant toute une gamme de tonalités à la transparence. Hood (2007) précise que plusieurs comportements réfrènent la possibilité d'une réelle transparence de l'appareil étatique. Ces comportements " pudiques " et donc limitant la transparence sont motivés sur l'évitement de blâmes. Il est politiquement plus rentable d'éviter des blâmes que de compter sur des réussites et l'électeur se souvient des mauvais coups et oublie facilement les bons! 
That is why a number of widely observed but often-criticized behavioural patterns in public management seem to constitute a set of agency, policy and presentational strategies that lie precisely on this troubled frontier territory between the force of transparency and the apparent imperative of blame-avoidance (Hood, 2007, p. 207).

Selon Hood (2007), la transparence gouvernementale s'exerce principalement par des moyens directs lorsqu'une communication s'établit entre le gouvernement et les citoyens ou par des moyens indirects lorsque le gouvernement et l'administration publique communiquent à l'aide d'une reddition de comptes toujours fort commentée par des experts et parfois certifiée par des auditeurs.

Au cours des quarante dernières années, la transparence indirecte a dominé et a servi principalement à rendre compte selon les perspectives des gouvernements au pouvoir. L'enjeu démocratique d'un plus grand degré de transparence se joue sur le plan de la participation des citoyens qui se doivent d'être éclairés par l'apport essentiel d'une information de qualité transmise en mode interactive permettant un échange et un dialogue. Pour cela, la transparence gouvernementale doit s'exercer au moyen de mécanismes directs.

Pour construire ou maintenir la confiance entre les acteurs, il est nécessaire de recourir à des mécanismes objectifs tels des processus d'audit, d'enquête ou d'évaluation qui mettent l'accent sur les faits. La déclaration du président Obama citée précédemment souligne l'importance pour un gouvernement de s'assurer de la confiance du public. Paquet (2011) établit un lien direct entre la confiance et la productivité : "Plus il y a de confiance entre les partenaires, plus la productivité augmente. Plus il y a de friction, moins la confiance règne, plus c'est la multiplication des griefs et l'improductivité » (Paquet, 2011, p. 87).

Afin de maintenir la cohésion, on établit des systèmes et des processus qui deviennent des règles à suivre. Les acteurs sociaux et politiques apprennent à adapter leurs messages aux circonstances et ainsi à donner les réponses attendues. Le député doit respecter la ligne du parti et les gestionnaires publics doivent se conformer aux politiques, aux procédures et aux bonnes pratiques de gestion généralement reconnues. La société fonctionne grâce à un ensemble de systèmes. À titre d'exemple, lorsque nous conduisons une automobile, nous traversons une intersection en relative confiance lorsque le feu de circulation est vert. La codification de notre environnement assure une prédictibilité et met en place des systèmes de confiance basés sur celle-ci. La règle de droit est un exemple de codification qui favorise par divers mécanismes l'édification de sociétés démocratiques.

L'audit gouvernemental aide au maintien et à l'adaptation des systèmes de confiance, notamment lors de la reddition de comptes découlant de la délégation de pouvoir et de responsabilité à l'intérieur de l'appareil gouvernemental. Malgré la portée des mandats (audit financier, de conformité, d'optimisation des ressources ou de performance), l'apport de l'audit est fortement axé sur la conformité à des lois, des règlements, des politiques, des procédures ou sur la mise en place de bonnes pratiques de gestion. La fonction d'audit permet notamment de veiller à la conformité aux règles et, telle une auto-patrouille sur l'autoroute, elle permet aussi de dissuader les écarts de conduite. L'audit exerce également une fonction d'introspection qui agit comme un possible catalyseur de changement permettant 
aux acteurs de gouverne d'adapter les systèmes et les règles lorsque ceux-ci n'apportent pas de valeur ajoutée pour atteindre les objectifs. Les systèmes ne se régulant pas eux-mêmes, par la dynamique de la gouvernance collaborative les acteurs préservent la discrétion de faire évoluer les règles du jeu.

Les auditeurs ont très rarement l'occasion de sortir à l'extérieur de l'enclos des règles et des processus établis. S'ils sont de précieux collaborateurs pour améliorer les systèmes de confiance (règles et processus), la prérogative de l'innovation permettant notamment de modifier la vision et les objectifs demeure toujours entre les mains des acteurs politiques qui contrôlent les pouvoirs exécutif et législatif. Ce confinement de la fonction d'audit amoindrit dans une certaine mesure son indépendance et son objectivité. C'est ce que Radcliffe (2008) appelle le pragmatisme de la fonction d'audit. Selon lui, ce pragmatisme fort de l'acculturation des auditeurs à leur environnement entraîne deux conséquences. La première est que la fonction d'audit participe au maintien des secrets de l'appareil d'État et la seconde a trait à l'autocensure pratiquée par les auditeurs quant à ce qui peut être divulgué au public. En fait, la fonction d'audit prend part à la transparence que certains qualifient d'opaque, car elle est le fruit de la reddition de comptes effectuée selon les perspectives de l'appareil d'État bureaucratique qui est offerte au public en mode unidirectionnel.

La mise en place de systèmes auxquels les acteurs doivent se conformer pour assurer la cohésion constitue la stratégie " push " de la confiance. Cette stratégie met l'accent sur les dimensions extrinsèques à l'acteur lui demandant de respecter les règles du jeu, respect qui engendre la prédictibilité et par conséquent la confiance au système. La fonction d'audit tient le rôle d'agent de conformité dans ces systèmes. Poussée à l'extrême, cette stratégie risque toutefois de donner naissance à un "État policier " au sein duquel les citoyens sont appelés à devenir de petits robots sans initiative ni créativité.

La confiance en des systèmes renvoie davantage au concept de confidence dans la langue anglaise, alors que celui de trust est lié aux dimensions relationnelles entre les acteurs. Si les citoyens accordent leur confiance aux systèmes, on ne peut en dire autant de la confiance donnée à leurs leaders politiques; la confiance relationnelle s'érode dans nos sociétés. L'appât du gain, l'accumulation du capital et la célébrité sont devenus des fins en soi nous transformant en acteurs socioéconomiques qui jouent le jeu des systèmes. Les relations s'établissent par l'expérimentation et les échanges entre des personnes. Il est plus aisé de faire confiance à une relation bien établie forte d'un historique sans accroc.

De Saint-Exupéry nous rappelle la signification du mot " apprivoiser ». Lorsque le renard rencontre le petit prince, aucune confiance n'existe entre eux. À la suite d'un processus permettant de s'apprivoiser, une relation naît et la confiance devient possible. "On ne connaît que les choses que l'on apprivoise, dit le renard. Les hommes n'ont plus le temps de rien connaître. Ils achètent des choses toutes faites chez les marchands. Mais comme il n'existe point de marchands d'amis, les hommes n'ont plus d'amis. Si tu veux un ami, apprivoise-moi ! (De Saint-Exupéry, 2000, chap. 21). On ne peut donc mettre de côté la dimension relationnelle entre les acteurs qui constitue la stratégie " pull " de la confiance, car elle favorise la 
création de valeurs d'intégrité et de respect en faisant appel aux dimensions intrinsèques de l'être humain, véritable terreau de la créativité et de l'innovation. Pour innover, il faut nécessairement sortir de la tranchée et avoir assez de confiance et d'appui pour prendre des risques et apporter une réponse inattendue permettant l'innovation. La confiance relationnelle est fondée sur un mode interactif et déborde ainsi largement de la confiance en des systèmes de règles. Selon Lyman (2012), cette confiance compte trois ingrédients essentiels : la crédibilité, le respect et le sens de l'équité. Tout leader possède à des degrés divers une dose de ces trois ingrédients aux yeux de ses pairs. Un leader politique doit développer la stratégie " pull » et permettre aux citoyens ou aux autres parties prenantes de participer et d'influencer :

Trustworthy Leaders openly share information with people to help them participate in and influence the life of the organization. They invite people into discussions that will support the expansion of the organization's products and services. These leaders know that employees' contributions will be magnified to the degree that they have access to useful information. (Lyman, 2012, p. 12)

La codification par des règles et des normes à respecter représente le mortier de la maçonnerie sociétale. Toutefois, sans intégrité des acteurs, il est vain et illusoire de croire que la codification et les diverses mesures de contrôle permettent une entière confiance relationnelle. Conséquemment, l'adhésion aux valeurs, au projet de société et au contrat social s'en trouve amoindrie. Armstrong (2005, p. 3) traduit ce préalable en ces termes : "The integrity, transparency and accountability of public administrations are a prerequisite to and underpin public trust, as a keystone of good governance."

Les valeurs d'éthique demeurent la base fondamentale sur laquelle il est possible de construire. Sans la confiance du public basée sur les valeurs de respect et d'intégrité, les administrations publiques auront beau fixer toutes sortes de règles, elles risquent fort d'aller à la dérive notamment par de l'évasion fiscale et de l'économie au noir.

\section{— UN GOUVERNEMENT OUVERT : LE FRUIT D'UN DÉLICAT DOSAGE}

Une pleine transparence peut équivaloir à être à nu, à être dans un état où tout est au vu et au su de tous. Certes, personne ne s'attend à ce que les organisations ou les acteurs soient pleinement transparents, mais une meilleure transparence de la part des gouvernements ou des grandes entreprises est fréquemment réclamée. En fait, exiger davantage de transparence signifie en quelque sorte demander une plus grande responsabilisation, ce qui devrait se traduire par une hausse de la reddition de comptes sur le respect des engagements et des responsabilités et sur l'atteinte des résultats.

La vie en société établit tacitement le degré de transparence considéré comme acceptable et normal. Ainsi, il apparaît plus acceptable qu'une entreprise privée limite l'information sur ses activités commerciales selon un niveau de transparence qui, par ailleurs, serait jugé inacceptable pour un gouvernement. Or les enjeux environnementaux ramènent à l'avant-plan le fait que nous partageons un espace 
qui est du domaine public dans plusieurs de ses dimensions. Lorsque des deniers publics sont mis à contribution pour réaliser des activités, les citoyens s'attendent à un degré de transparence plus élevé. Inévitablement, les organisations, qu'elles soient du secteur privé ou public, ont l'obligation de rendre des comptes à des parties prenantes : propriétaires, actionnaires, citoyens, législateurs, etc. L'écart des attentes de transparence entre les secteurs public et privé se confirme dans l'application des lois d'accès à l'information et de protection des renseignements personnels. En règle générale, les lois d'accès à l'information s'appliquent au secteur public, tandis que les lois sur la protection des renseignements personnels sont davantage d'application universelle puisqu'elles ont comme objectif de protéger la vie privée. Les personnes morales que constituent les entreprises privées tiennent à protéger leur vie privée. Même les États et les administrations publiques ont leurs jardins secrets, dont ceux liés aux domaines de la sécurité ou de la compétitivité nationale. Un dosage est donc de mise pour maintenir une décence acceptable entre une culture d'ouverture totale et une culture du secret à outrance.

D'une part, les fervents de la culture du secret font valoir que leurs intérêts privés ne concernent personnes d'autres. Poussés à l'extrême, ils sont pour des comptes bancaires totalement privés et anonymes et pour la protection de leurs technologies et de leur savoir-faire tout en limitant au maximum toute forme de reddition de comptes, sauf celle à l'égard de leurs subalternes. Ce sont les monarques des temps modernes et pour eux la ressource " information " est un capital à préserver. Kantrowitz (1989) mentionne que la culture du secret engendre un environnement propice à la corruption et favorise une dynamique de division :

Corruption is a progressive disease. It diffuses from person to person across society by direct observations of its efficacy and its safety. The efficacy of the abuse of secrecy for interagency rivalry and for personal advancement is well illustrated by the array of abuses listed in Sec. 1.6(a). The safety of the abuse of secrecy for the abuser is dependent upon the enforcement of the Section. As abuses spread and become the norm, enforceability declines and corruption diffuses more rapidly.

Reagan's 1982 Executive Order \#12356 on National Security \{Sec. 1.6(a)\}: "In no case shall information be classified in order to conceal violations of law, inefficiency, or administrative error; to prevent embarrassment to a person, organization or agency; to restrain competition; or to prevent or delay the release of information that does not require protection in the interest of national security". (Kantrowitz, 1989)

D'autre part, pour les tenants de la culture d'ouverture, toutes les informations sont d'intérêt public et la libre circulation de celles-ci favorise la saine compétition et la démocratie. À l'extrême, la totale transparence peut aussi vouloir dire la totale surveillance. Il n'y aurait plus de liberté individuelle, car tout serait du domaine public; la planète deviendrait alors un grand village où tout est connu de tous ! Toutefois, les défenseurs de la liberté individuelle militent pour la préservation de la vie privée afin de sauvegarder des avantages concurrentiels dans une économie de marché compétitive. De plus, plusieurs renseignements ne sont pas pertinents pour la gestion du bien commun, ils relèvent exclusivement du domaine privé. D'un point de vue sociétal, les avantages de l'ouverture semblent plus importants 
que ses inconvénients. Dès lors que les citoyens estiment qu'une activité a des incidences dans le domaine public, il se crée alors des attentes de transparence plus élevées. Le phare qui devrait guider le degré de transparence est la préservation du bien commun et des intérêts des citoyens assurant la pérennité des acquis, notamment un environnement sain. Les questions environnementales ont mis en relief l'importance d'une plus grande responsabilité sociale des entreprises à l'égard de la préservation des biens publics. La démarcation entre ce qui est du domaine public et ce qui est du domaine privé n'est pas facile à tracer et rend difficile le dosage du degré de transparence.

L'information permet de moduler la transparence. Par analogie, on peut dire que l'information constitue la fibre façonnant les vêtements des acteurs sociaux : nos institutions, gouvernements, administrations publiques ou grandes entreprises sont tous habillés à la mode vestimentaire de notre époque, mode qui évolue en fonction des intérêts et des enjeux des divers acteurs sociaux. L'exploitation des ressources naturelles est l'un des enjeux actuels qui soulèvent des débats de société sur la conciliation du développement économique et de la préservation des milieux naturels. Pensons notamment au développement du Grand Nord, aux sables bitumineux, aux gaz de schiste ou aux réserves de pétrole du golf du SaintLaurent. Les citoyens ont besoin d'être rassurés par des audits, des enquêtes ou des évaluations qui viennent supporter la confiance sur les informations fournies.

Un gouvernement ouvert ne devrait pas donner accès à l'information uniquement lorsqu'un citoyen en fait la demande, tel qu'il est actuellement prévu par les lois d'accès à l'information. En fait, il y a une gradation du caractère « ouvert » d'un gouvernement. L'étape suivante consiste à rendre accessibles sur le Web les informations gouvernementales de façon proactive. À titre d'exemple, Gautrin (2012) préconise ce qui suit pour le gouvernement du Québec :

La divulgation proactive de tout ce qui pourrait être accessible à la demande d'un citoyen en est l'un des éléments clés. Il y aurait lieu d'étendre davantage cette divulgation à toutes les données statistiques, aux rapports d'experts ou aux autres documents obtenus grâce à des fonds publics (contrats, subventions, etc.) qui ont permis l'établissement d'une position gouvernementale. (Gautrin, 2012, p. 68)

La divulgation proactive de l'information gouvernementale favorise une communication interactive avec les citoyens. Certes, cela exige un profond changement de culture puisque nos bureaucraties d'État sont souvent refermées sur elles-mêmes et ne suscitent guère la participation et la collaboration des citoyens. Sans ouverture, il ne peut y avoir de réelle participation et de collaboration des citoyens. Dans ce contexte, la transparence ressemble davantage à un masque et il subsiste encore une grande distance entre le petit prince et le renard, car le processus d'apprivoisement ne se fait pas. Le citoyen devient un client qui est servi selon les termes de la bureaucratie.

La mise en commun d'un tableau de bord gouvernemental sur le Web rendant accessibles les informations d'intérêt public pour l'ensemble des services gouvernementaux permettrait de resserrer les liens de confiance entre les citoyens et leurs institutions publiques, leurs députés et leurs ministres. Ce tableau de bord offrirait également une vision du chemin parcouru et des possibilités. 


\section{- LA PARTICIPATION ET LA COLLABORATION CITOYENNES : UNE DÉMOCRATIE AVEC UN GRAND D}

Les moyens techniques de notre époque sont extraordinaires. Les technologies de l'information, la statistique et les connaissances scientifiques nous offrent des possibilités de réaliser des œuvres humanitaires sans pareil. Mars est aujourd'hui accessible alors que la persistance d'immenses bidonvilles sur notre planète demeure un grand paradoxe. Le champ des possibilités est ouvert, mais l'exercice politique constitue l'arène qui le limite souvent.

Encore aujourd'hui, notre régime parlementaire britannique maintient un modèle relationnel de l'exercice de l'autorité politique selon une approche hiérarchique axée du haut vers le bas. Ce système d'inspiration monarchique transforme la grande majorité d'entre nous en sujets. Le caractère participatif et démocratique de notre régime parlementaire est embryonnaire et pourrait être grandement bonifié par l'apport des technologies de l'information du XXI ${ }^{\mathrm{e}}$ siècle.

Il est possible de croire qu'au cours des prochaines années chaque citoyen aura un compte réseau gouvernemental sécurisé avec une authentification fiable. L'ensemble de ces comptes réseau pourrait par la suite servir à créer des "réseaux politiques " par comtés, par régions, par provinces, par pays et par continents. Ces réseaux locaux, régionaux, nationaux et internationaux feraient la promotion de la citoyenneté et favoriseraient l'interaction entre les citoyens et leurs représentants politiques, leurs gouvernements et leurs administrations publiques. Si nos moteurs carburent véritablement à l'énergie démocratique alors pourquoi se priver des nouvelles technologies plus performantes pour atteindre nos objectifs? En fait, ces " réseaux politiques " peuvent donner aux citoyens les moyens de jouer leur rôle et de mieux assumer leurs responsabilités. Le citoyen devient alors de facto une partie prenante participative à part entière de la gouvernance collaborative.

Si l'on donnait aux citoyens le pouvoir d'initiative, ils pourraient exiger des réponses à des questions de fond. Ce pouvoir établi dans un cadre d'application précis permettrait de tenir ou de réaliser des référendums, des commissions d'enquête publiques, des mandats précis d'audit ou des évaluations de programme gouvernemental, ou bien de proposer ou de modifier des lois. La fonction d'audit de la gouvernance pourrait alors jouer un rôle de support aux initiatives populaires des citoyens. Comme l'écrit Larocque :

Dans son principe même, l'initiative populaire est au cœur de la démocratie puisqu'elle affirme la seule souveraineté démocratique possible, celle du peuple. Par sa pratique, l'initiative populaire permet au peuple de maintenir un contrôle sur le pouvoir qui est le sien. Il ne suffit pas qu'une constitution établisse explicitement la souveraineté populaire, il faut que cette souveraineté s'incarne dans des dispositions permettant de l'exercer concrètement. (Larocque, 2006, p. 42)

Aucun système n'est parfait. Il est certes nécessaire d'avoir des leaders, mais les itinéraires pourraient être davantage discutés et décidés démocratiquement avec la participation et la collaboration continue des citoyens. La démocratie participative avec les technologies de l'information est du domaine du possible, mais elle ne peut s'enraciner et s'épanouir que par un changement culturel important. 
À la suite d'une élection, le programme demeure sous le contrôle strict du parti politique au pouvoir. Aucun mécanisme ne permet aux citoyens de lancer directement des débats en les mettant à l'ordre du jour du programme. Pourtant, avec les nouvelles technologies de l'information, il est aussi facile de tenir des référendums sur de grandes questions de société, telle l'exploitation des gaz de schistes, que de conduire un sondage pour cerner l'effet sur la popularité du parti au pouvoir de sa dernière stratégie de communication. Il importe ici de nuancer. Nos modèles de gouvernance prévoient un pouvoir à connotation législative (grandes orientations et surveillance stratégique des grands enjeux et risques d'entreprise) et un pouvoir exécutif. Il ne s'agit pas d'éliminer le pouvoir exécutif qui a comme fonction d'orchestrer les ressources afin d'atteindre les objectifs, mais bien de se doter de mécanismes pour veiller à ce que le pouvoir législatif repose véritablement entre les mains de la majorité de ceux qui ont délégué leur pouvoir, soit les actionnaires dans le cas d'une entreprise privée ou les citoyens dans le cas d'un État.

Les citoyens assistent à une prolifération de l'information sur le Web. Pour décoder et interpréter cette information, il est important de créer des forums de discussion interactifs pour débattre d'idées et participer à la vie démocratique de notre société. C'est pourquoi l'ouverture des gouvernements devrait se faire autant sur le front de la disponibilité de l'information que sur celui de la communication interactive avec les citoyens.

\section{- L'OBLIGATION DE RENDRE COMPTE : AU SERVICE DE L'EFFICACITÉ ET DE L'EFFICIENCE}

Les gouvernements et les administrations publiques ont déployé des efforts considérables pour atteindre des résultats et améliorer leur rendement : systèmes de programmation budgétaire et de prix de revient, processus de planification stratégique et de mesure de la performance, déclarations de services aux citoyens, cadre de responsabilisation de gestion, rapports annuels de performance, etc. De plus, les agents du Parlement rendent publics les rapports qui permettent d'apprécier l'application de lois spécifiques ou le rendement d'activités gouvernementales, comme les rapports du vérificateur général, du commissaire à l'environnement, du commissaire aux langues officielles ou du commissaire à l'accès à l'information. Devant l'ampleur des activités et les nombreux rapports faisant état de la performance de l'administration publique, le citoyen est pris au dépourvu et il lui est difficile de bien voir la situation ou même d'appréhender dans toute sa complexité cet amoncèlement d'information. De surcroît, cette divulgation d'information se fait selon une dynamique unidirectionnelle et donc par le truchement d'une reddition de comptes établie selon la vision et les référents de l'appareil d'État.

La divulgation proactive de l'information gouvernementale sur le Web intégrée à un tableau de bord procurerait d'une part une vision du travail accompli et de la route à parcourir et améliorerait d'autre part les possibilités de participation des citoyens. Toutefois, pour qu'une véritable communication interactive s'établisse, il faudrait outiller les citoyens de capacités d'audit, d'évaluation et d'enquête. Le principe est fort simple : il faut offrir aux parties en communication interactive 
les mêmes outils d'analyse et l'accès aux mêmes droits et expertises. L'audit de la gouvernance pourrait offrir une garantie sur l'intégrité des systèmes et sur la fiabilité de l'information sur la performance communiquée en mode interactif sur une base périodique.

La responsabilisation et l'obligation de rendre compte exigent plus qu'un simple accès aux informations. Selon Fox (2005, p. 665) : "If the power of transparency is based on the "power of shame", then its influence over the really shameless could be quite limited. It turns out that transparency is necessary but far from sufficient to produce accountability ". Fox met en relief deux aspects importants du concept d' "accountability ». Premièrement, pour être effective, cette obligation de rendre compte doit se baser sur le droit d'exiger des comptes, ce que Fox appelle l'« answerability ». Deuxièmement, il est nécessaire de pouvoir non seulement distribuer des bonis à la performance, mais également infliger des sanctions, ce que Fox appelle la " hard accountability ". Il conclut qu'il ne faut pas placer la barre trop haute en ce qui a trait à la capacité de la transparence pour obtenir une reddition de comptes effective :

One should not expect answerability from opaque transparency, and one should not expect hard accountability from answerability. To take the next step and address hard accountability would involve going beyond the limits of transparency and dealing with both the nature of the governing regime and civil society's capacity to encourage the institutions of public accountability to do their job. (Fox, 2005, p. 669)

La mise en place de dispositions d'initiative populaire serait utile au raffermissement du droit d'exiger des comptes selon les perspectives des citoyens et elle serait garante d'une meilleure reddition de comptes sur l'efficience, l'efficacité, l'économie, l'éthique, l'environnement, etc. Meijer et ses collègues définissent un gouvernement ouvert en ces termes : "L'ouverture de l'État est la mesure dans laquelle les citoyens peuvent surveiller et influencer les processus gouvernementaux grâce à la possibilité qui leur est offerte d'accéder à l'information gouvernementale et aux instances décisionnelles " (Meijer, Curtin et Hillebrandt, 2012, p. 17).

Comme mentionné précédemment, l'audit sert à maintenir ou à raffermir le lien de confiance entre l'émetteur et le récepteur de la reddition de comptes. L'obligation de rendre des comptes est la contrepartie de la délégation de pouvoir. Des liens de confiance doivent être maintenus ou raffermis à chacun des paliers de délégation, soit entre les citoyens et le Parlement (les députés); entre le Parlement (les députés - pouvoir législatif) et le gouvernement (le cabinet ministériel - pouvoir exécutif); entre le gouvernement et les administrateurs publics; entre les administrateurs d'État et les gestionnaires publics exécutifs.

En ce qui concerne le premier niveau de délégation, celui entre les citoyens et leurs représentants formant le Parlement, les élections, mécanisme fondamental de notre démocratie, sont pour l'instant l'unique dispositif direct par lequel le citoyen pose un geste de participation à la vie politique. Sans mécanisme de suivi ouvert et continu qui permet d'apprécier la performance du travail de représentation des citoyens par les élus, nous ne pouvons que présumer de la qualité de notre vie démocratique. La mise en place de mécanismes de communication interactive des citoyens avec leurs élus, leur gouvernement, leur administration publique n'a pas pour fonction de se substituer à nos institutions. Ces instruments favorisent 
une plus grande transparence et une meilleure efficacité et efficience quant à la gestion du bien commun et à la prise en compte des intérêts des citoyens par nos institutions. Pour que la fonction d'audit soutienne la gouvernance, il faut qu'elle soit mise à contribution pour offrir directement des services d'assurance aux citoyens ou aux actionnaires, car ceux-ci sont les délégateurs de première instance du pouvoir et des responsabilités.

\section{- CONCLUSION : L'AUDIT DE LA GOUVERNANCE EN DEVENIR}

Il subsistera toujours un écart entre les principes, les objectifs ou les intentions et leur concrétisation dans la pratique. La déclaration du président Obama citée dans l'introduction du présent texte fait appel à plusieurs principes et objectifs : haut degré d'ouverture du gouvernement, confiance du public, système de transparence, de participation publique et de collaboration, raffermissement de la démocratie, promotion de l'efficience et de l'efficacité. Pour réaliser ces principes, il est primordial d'établir un dialogue interactif entre les citoyens, leurs institutions publiques et les acteurs politiques. La fonction d'audit peut favoriser ce dialogue en contribuant par des états de situation les plus objectifs possibles au maintien ou au raffermissement de la confiance des citoyens.

Ce texte a présenté succinctement la fonction d'introspection des institutions publiques qui prend la forme d'audit, d'évaluation, d'enquête ou d'inspection des activités, fonction qui certifie les diverses formes de reddition de comptes. Il a fait ressortir l'importance de la confiance comme préalable à la cohésion et à l'innovation de nos sociétés et a amorcé une réflexion sur l'émergence d'une plus grande attente de transparence à l'égard de la gestion du bien commun suscitée par l'apport des technologies de l'information à la communication interactive. Enfin, il soulève des questionnements sur l'utilisation de moyens techniques et scientifiques pour raffermir la démocratie à l'ère où le village global de Marshall McLuhan est devenu une réalité indubitable.

Il serait présomptueux de croire que la fonction d'audit est la locomotive qui servira à concrétiser les principes et les objectifs évoqués dans la déclaration d'Obama. Toutefois, faire fi d'objectivité et d'indépendance, c'est renoncer à une vision claire de nos actions et de notre devenir. La globalisation induit la gouvernance collaborative qui a l'énorme défi de maintenir une coordination satisfaisante de la pluralité des acteurs possédant chacun leur dose d'ingrédients du pouvoir, soit la force du nombre, l'information, le savoir, le capital naturel et financier et la force militaire ou coercitive. "Dans un monde d'organisations où les ressources, le pouvoir, l'information et les connaissances sont distribués entre plusieurs mains, personne ne peut plus imposer autocratiquement sa gouverne " (Paquet, 2011, p. 47).

L'audit gouvernemental contribue humblement à maintenir et à adapter, au besoin, les systèmes de confiance (stratégie push de la confiance), notamment lors de la reddition de comptes découlant de la délégation de pouvoir à l'intérieur de l'appareil d'État. La fonction d'audit pourrait également être mise à contribution pour favoriser le dialogue et la participation des acteurs d'une gouvernance collaborative. Dans une perspective d'ouverture sur le monde, l'audit de la gouvernance est en devenir. 


\section{BIBLIOGRAPHIE}

Anderson, C. H. (1837). Les habits neufs de l'empereur, http://feeclochette.chez.com/ Andersen/habitsneufs.htm (page consultée le 17 mai 2012).

Armstrong, E. (2005). Integrity, Transparency and Accountability in Public Administration: Recent Trends, Regional and International Developments and Emerging Issues, Nations Unies.

De Saint-Exupéry, A. (2000). Le Petit Prince, Paris, Gallimard.

Fox, J. (2007). «The Uncertain Relationship Between Transparency and Accountability ", Development in Practice, vol. 17, $n^{\circ}$ 4, p. 663-671.

Gautrin, H.F. (2012). Gouverner ensemble : comment le Web 2.0 améliora-t-il les services aux citoyens, Québec, Bibliothèque et Archives nationales du Québec.

Hood, C. (2007). "What Happens When Transparency Meets Blame-avoidance ", Public Management Review, vol. 9, n² 2, p. 191-210.

Kantrowitz, A. (1989). "The Weapon of Openness ", Foresight Background, n 4, rev. 0.

Larocque, A. (2006). Au pouvoir, Citoyens! Mettre fin à l'usurpation des partis politiques, Montréal, Éditions BLG.

Lyman, A. (2012). The Trustworthy Leader, San Francisco, Jossey-Bass.

McLuhan, M. (1969). War and Peace in the Global Village, New York, Bantam.

Meijer, A. J., D. Curtin et M. Hillebrandt (2012). « La gouvernance ouverte : relier visibilité et moyens d'expression ", Revue internationale des sciences administratives, vol. 78, $\mathrm{n}^{\circ} 1, \mathrm{p} .13-32$.

Obama, B. (2009). Transparency and Open Government: Memorandum for the Heads of Executive Departments and Agencies, www.gpo.gov/fdsys/pkg/FR-2009-01-26/pdf/E9-1777.pdf (page consultée le 3 mai 2012).

Open Government Partnership (2011). Déclaration de gouvernement transparent, www. opengovpartnership.org/d\%C3\%A9claration-de-gouvernement-transparent (page consultée le 3 mai 2012).

Paquet, G. (2011). Gouvernance collaborative : un antimanuel, Montréal, Liber.

Radcliffe, V. S. (2008). « Public Secrecy in Auditing: What Government Auditors cannot Know ", Critical Perspectives on Accounting, vol. 19, n 1, p. 99-126.

Riopel, M. (2009). Épistémologie et enseignement des sciences, https://sites.google.com/site/ epistemologieenseignement/ (page consultée le 15 août 2012). 